\title{
Integrated Pest Management System with Impulsive Control of Spatial Heterogeneity
}

\author{
ZHOU Hongling ${ }^{1}$, SHEN Lin ${ }^{1,2, *}$ and WANG Shu ${ }^{2}$ \\ 1 School of Mathematics and Statistics, Huanghuai University, Zhumadian 463000, \\ China. \\ 2 School of Mathematics, Faculty of Science, Beijing University of Technology, Ping \\ Le Yuan 100, Beijing 100124, China.
}

Received 24 December 2020; Accepted 3 May 2021

\begin{abstract}
An impulsive integrated pest management system with diffusion is investigated within this paper. The conditions for pest eradication of the impulsive system without natural enemies are established based on the Krein-Rutman theorem and the comparison principle for parabolic equations. Integrated pest management can be achieved at an exponential rate, when the principal eigenvalues of the auxiliary system is large enough. Numerical simulations are presented to demonstrate the theoretical results. A discussion is given at the end.
\end{abstract}

AMS Subject Classifications: 65Q10, 68Q85, 68U20

Chinese Library Classifications: O175.2, O29, O242

Key Words: Integrated pest management; eigenvalue problem; pest control; diffusion.

\section{Introduction}

The concept of integrated pest management (IPM) was introduced in the late 1950s and was widely practised during the 1970s and 1980s [1-4]. It is defined as a process consisting of the balanced use of all the pest controls that are environmentally compatible, economically feasible, and socially acceptable to reduce pest populations to tolerable levels. And pest control often involves biological, cultural, and chemical control [1-6].

Biological control, which is defined as the suppression or the elimination of pest populations by natural enemies, has been an important aspect in an IPM strategy [6]. Natural

${ }^{*}$ Corresponding author. Email addresses: 8210s@163.com (H. L. Zhou), 8810s@163.com (L. Shen), wangshu@ bjut.edu.cn (S. Wang) 
enemies used for biological control can be divided into three categories: Predatory enemies, including lacewings, ladybugs and many insectivorous birds; Parasitic enemies, including parasitic wasps, parasitic flies, etc; The pathogenic microorganism, including the bacterium, bacillus thuringiensis, and so on. This control strategy is usually used by releasing natural enemies at a critical time to reduce a pest's population $[5,7,8]$. Biological control has been applied to greenhouse culture, the use of Encarsia formosa against Trialeurodes vaporariorum on tomatoes and cucumbers is one of the first successful cases of biological control in greenhouses [3,4,9]. Another important method for pest control is chemical control. In most cropping systems, pesticide are still the principal means of controlling pests. They can be cheap and are easy to apply, act fast [5], but chemical control also has certain harmfulness, pesticide can not only environmental pollution, but also cause plant phytotoxicity and waste of resources, and if incorrectly used, also easily lead to human and animal poisoning.

In mid 80s, there has been renewed interest in modelling IPM. Many IPM strategies such as releases of natural enemies at critical times and killing pests instantly by spraying pesticides have been proposed by mathematical models [6-16]. In [7], Liang et al. developed two novel pest-natural enemy interaction models incorporating the evolution of pesticide resistance, they investigated the number of natural enemies to be released when threshold conditions for the extinction of the pest population in two different control tactics are reached. In [16], Tang et al. modelled IPM including residual effects of pesticides in terms of fixed pulse-type actions.

However, the aforementioned above studies ignore the effect of space. The questions that arise are: in the case of uneven distribution of space, if we aim to eradicate the pest, how do we release the natural enemies? what proportion do we need to kill the pests by pesticide? To address these questions, we present an impulsive differential equation with diffusion. In the established model, (a) insect and natural enemies are all dealt with control in space; (b) distributed control of pest are considered by a combination of the action of natural enemies and impulsive control which include pesticide spraying and natural enemies releasing; (c) because of the pesticide's impact on the natural enemies, a proportion of the natural enemies could be killed at the time the pesticide is sprayed to kill the pests [17-19]. When we ignore the effect of space, the established model can be found in many domain of the applied sciences [20-22].

The aim of this work is to determine the conditions for pest eradication by pesticide spray-ing at critical times and the role of natural enemies releasing in enhancing the control. In order to show the conditions of pest extinction, Firstly, we discuss the model without natural enemies and get the sufficient and necessary conditions of pest extinction. Secondly we analyze the model without pest and we find the positive periodic solution of natural enemies. By using the conclusion of Section 3 and Section 4, we finally get the conditions for the extinction of the pests. Examples and numerical simulations are presented in Section 6 to illustrate the feasibility of our results. Section 7 involves some concluding remarks and discussions. 


\section{Two Species Model}

The densities of pests and natural enemies are denoted by $u(x, t)$ and $v(x, t)$, respectively. The habitat is denoted by $\Omega . u$ and $v$ are described by the following dynamical model :

$$
\begin{cases}\frac{\partial u}{\partial t}-d_{1} \frac{\partial^{2} u}{\partial x^{2}}=r u\left(1-\frac{u}{K}\right)-\frac{\beta u v}{1+\beta h u}, & x \in \Omega, t \in R^{+} \backslash n \tau, n \in N \\ \frac{\partial v}{\partial t}-d_{2} \frac{\partial^{2} v}{\partial x^{2}}=-\alpha v+\frac{\eta \beta u v}{1+\beta h u}, & x \in \Omega, t \in R^{+} \backslash n \tau, n \in N \\ u\left(x, n \tau^{+}\right)=\rho(x) u(x, n \tau), & x \in \Omega, n \in N \\ v\left(x, n \tau^{+}\right)=\rho_{0}(x) v(x, n \tau)+I(x), & x \in \Omega, n \in N \\ u(x, 0)=u_{0}(x), v(x, 0)=v_{0}(x), & x \in \Omega \\ u_{x}=v_{x}=0, & x \in \partial \Omega\end{cases}
$$

where $d_{1}, d_{2}$ denote diffusion rate of pests and natural enemies, respectively; $r$ represents growth rate of pests; $\alpha$ is mortality rate of natural enemies; $K$ is carrying capacity of pests; $\beta$ is encounter rate; $h$ represents handing time; $\eta$ denotes conversion rate; $\tau$ is the period of the integrated control.

Let $u_{0}(x), v_{0}(x)$ denote the initial density of pest population and natural enemies, respectively. The Neumann boundary condition holds for the two populations. At every period, pesticide is used to control pest population, the effectiveness of pesticides is different at different locations, and the pesticide will also affect natural enemies. $\rho(x), \rho_{0}(x)$ denote the survival rate of the pest and natural enemies after the $n$th pesticide, respectively. $I(x)$ represents the release function of the natural enemies at $n \tau$. We assume $u\left(x, n \tau^{+}\right), v\left(x, n \tau^{+}\right)$are the density of pests and natural enemies at the beginning of the $(n+1)$ th period, $u(x, n \tau), v(x, n \tau)$ are the density of pests and natural enemies at the end of the $n$th period. So, the pests and natural enemies at the beginning of the new period are denoted as follow

$$
\begin{aligned}
& u\left(x, n \tau^{+}\right)=\rho(x) u(x, n \tau), \\
& v\left(x, n \tau^{+}\right)=\rho_{0}(x) v(x, n \tau)+I(x) .
\end{aligned}
$$

Before the research start, the following hypotheses are postulated:

(H1) $\Omega \subset R$ is a bounded domain of length $l$ and

$$
l, r, K, \beta h, \alpha, \eta, \tau \in(0,+\infty), d_{1}, d_{2}, h \in[0,+\infty) .
$$

(H2) $\rho, \rho_{0}, I, u_{0}, v_{0} \in L^{\infty}(\Omega)$,

$$
\begin{array}{ll}
c \leq \rho(x) \leq 1, \quad c_{0} \leq \rho_{0}(x) \leq \rho_{0}^{*} \leq 1, & x \in \Omega, \\
I_{\min } \leq I(x) \leq I_{\max }, & x \in \Omega, \\
0 \leq u_{0}(x), \quad 0 \leq v_{0}(x), & x \in \Omega,
\end{array}
$$


where $c, c_{0}, \rho_{0}^{*} \in(0,1), I_{\min }, I_{\max } \in(0,+\infty)$.

We mention that the permanence and the existence of the unique periodic solution to (2.1) without the release function of the natural enemies are derived as in [23]. Please prepare your tex file. Thank you for your cooperation.

\section{Single species pest model}

The model without natural enemies population, but with a pesticide at the end of the period is represented as

$$
\begin{cases}\frac{\partial u}{\partial t}-d_{1} \frac{\partial^{2} u}{\partial x^{2}}=r u\left(1-\frac{u}{K}\right), & x \in \Omega, t \in R^{+} \backslash n \tau, \\ u\left(x, n \tau^{+}\right)=\rho(x) u(x, n \tau), & x \in \Omega, n \in N, \\ u(x, 0)=u_{0}(x), & x \in \Omega, \\ u_{x}=0, & x \in \partial \Omega .\end{cases}
$$

In order to give a necessary condition and sufficient condition for the eradication of population $u$. we need some basic properties of the following subsystems:

$$
\begin{aligned}
& \begin{cases}\frac{\partial \varphi}{\partial t}-d_{1} \frac{\partial^{2} \varphi}{\partial x^{2}}-r \varphi=\lambda \varphi, & x \in \Omega, t \in(0, \tau), \\
\varphi\left(x, 0^{+}\right)=\rho(x) \varphi(x, \tau), & x \in \Omega, \\
\varphi_{x}=0, & x \in \partial \Omega, t \in(0, \tau),\end{cases} \\
& \begin{cases}-\frac{\partial \Psi}{\partial t}-d_{1} \frac{\partial^{2} \Psi}{\partial x^{2}}-r \Psi=\lambda \Psi, & x \in \Omega, t \in(0, \tau), \\
\Psi(x, \tau)=\rho(x) \Psi\left(x, 0^{+}\right), & x \in \Omega, \\
\Psi_{x}=0, & x \in \partial \Omega, t \in(0, \tau) .\end{cases}
\end{aligned}
$$

The next Lemma states the existence of the principal eigenvalue and properties related to the eigenvalue problems (see [24]).

Lemma 3.1. (i) System (3.2) exists a principal eigenvalue $\lambda_{1}$, which is also the principal eigenvalue to the adjoined system (3.3);

(ii) there exist eigenvectors $\varphi_{1}$ to (3.2) and $\Psi_{1}$ to (3.3), corresponding to $\lambda_{1}$ and satisfying

$$
\varphi_{1}(x, t), \Psi_{1}(x, t) \geq c_{1}>0, \quad(x, t) \in \Omega \times(0, \tau) .
$$

Without loss of generality, we also represent the extension by T-periodicity of $\varphi_{1}$ and $\Psi_{1}$ by $\varphi_{1}$ and $\Psi_{1}$, respectively.

Theorem 3.1. Suppose $u_{0}$ satisfies condition (H2), (i) if the solution of (3.1) satisfies

$$
u(t) \rightarrow 0, \quad \text { in } L^{\infty}(\Omega) \text { as } t \rightarrow+\infty,
$$


then $\lambda_{1} \geq 0$.

(ii) if $\lambda_{1}>0$, then the solution $u$ to (3.1) satisfies $u(t) \rightarrow 0$, in $L^{\infty}(\Omega)$ as $t \rightarrow+\infty$.

Proof. (i) Assume $\lambda_{1}<0$. Then, for any $0<\varepsilon<-\lambda_{1}$, there exists $N(\varepsilon) \in N$ such that

$$
0 \leq \frac{r}{K} u(x, t)<\varepsilon
$$

in $\Omega \times(n \tau,(n+1) \tau)$, and for any $n \in N, n \geq N(\varepsilon)$. Thanks to the comparison result for parabolic equations [25], for any $n \geq N(\varepsilon)$, we deduce

$$
0 \leq \underline{u}(x, t) \leq u(x, t)
$$

in $\Omega \times(n \tau,(n+1) \tau)$, and for any $n \in N, n \geq N(\varepsilon)$, where $\underline{u}$ is the solution to

$$
\begin{cases}\frac{\partial \underline{u}}{\partial t}-d_{1} \frac{\partial^{2} \underline{u}}{\partial x^{2}}=r \underline{u}-\varepsilon \underline{u}, & x \in \Omega, t \in R^{+} \backslash n \tau, t>N(\varepsilon) \tau, \\ \underline{u}\left(x, n \tau^{+}\right)=\rho(x) \underline{u}(x, n \tau), & x \in \Omega, t \in R^{+} \backslash n \tau, t>N(\varepsilon) \tau, \\ \underline{u}_{x}=0, & x \in \partial \Omega, t \in R^{+} \backslash n \tau, t>N(\varepsilon) \tau, \\ \underline{u}\left(x, N(\varepsilon) \tau^{+}\right)=u\left(x, N(\varepsilon) \tau^{+}\right), & x \in \Omega .\end{cases}
$$

Since $\lim _{t \rightarrow \infty} u(t)=0$ in $L^{\infty}(\Omega)$, we have

$$
\underline{u}(t) \rightarrow 0 \text {, in } L^{\infty}(\Omega) \text {, as } t \rightarrow+\infty .
$$

Multiply the first equation in (3.4) by $\Psi_{1}$, integrate over $\Omega \times(n \tau,(n+1) \tau)$, and recall (3.3) to discover

$$
\begin{aligned}
& \int_{\Omega} \underline{u}(x,(n+1) \tau) \Psi_{1}(x,(n+1) \tau) \mathrm{d} x-\int_{\Omega} \underline{u}\left(x, n \tau^{+}\right) \Psi_{1}\left(x, n \tau^{+}\right) \mathrm{d} x \\
= & \int_{n \tau}^{(n+1) \tau} \int_{\Omega} \underline{u}\left[\frac{\partial \Psi_{1}}{\partial t}+d_{1} \frac{\partial^{2} \Psi_{1}}{\partial x^{2}}+r \Psi_{1}-\varepsilon \Psi_{1}\right] \mathrm{d} x \mathrm{~d} t .
\end{aligned}
$$

According to (3.3) and (3.4), we deduce

$$
\begin{aligned}
& \int_{\Omega} \underline{u}\left(x,(n+1) \tau^{+}\right) \Psi_{1}\left(x, 0^{+}\right) \mathrm{d} x-\int_{\Omega} \underline{u}\left(x, n \tau^{+}\right) \Psi_{1}\left(x, 0^{+}\right) \mathrm{d} x \\
= & -\left(\lambda_{1}+\varepsilon\right) \int_{n \tau}^{(n+1) \tau} \int_{\Omega} \underline{u}(x, t) \Psi_{1} \mathrm{~d} x \mathrm{~d} t>0 .
\end{aligned}
$$

This implies that $\left(\int_{\Omega} \underline{u}\left(x, n \tau^{+}\right) \Psi_{1}\left(x, 0^{+}\right) \mathrm{d} x\right)_{n}$ is an increasing sequence for $n \geq N(\varepsilon)$. Therefore, it is not converge to 0 . Thus, we get a contradiction, and the conclusion is that $\lambda_{1} \geq 0$.

(ii) Assume $\lambda_{1}>0$. Thanks to the comparison result for parabolic equations [25,26], we have

$$
0 \leq u(x, t) \leq \bar{u}(x, t)
$$


in $\Omega \times[0,+\infty)$, where $\bar{u}$ is the solution to

$$
\begin{cases}\frac{\partial \bar{u}}{\partial t}-d_{1} \frac{\partial^{2} \bar{u}}{\partial x^{2}}=r \bar{u}, & x \in \Omega, t \in R^{+} \backslash n \tau, \\ \bar{u}\left(x, n \tau^{+}\right)=\rho(x) \bar{u}(x, n \tau), & x \in \Omega, n \in N, \\ \bar{u}_{x}=0, & x \in \partial \Omega, t \in R^{+} \backslash n \tau, \\ \bar{u}(x, 0)=u(x, 0), & x \in \Omega .\end{cases}
$$

Multiply the first equation in (3.5) by $\Psi_{1}$ and integrate over $\Omega \times(n \tau,(n+1) \tau)$, we deduce that

$$
\begin{aligned}
& \int_{\Omega} \bar{u}\left(x,(n+1) \tau^{+}\right) \Psi_{1}\left(x, 0^{+}\right) \mathrm{d} x-\int_{\Omega} \bar{u}\left(x, n \tau^{+}\right) \Psi_{1}\left(x, 0^{+}\right) \mathrm{d} x \\
= & -\lambda_{1} \int_{n \tau}^{(n+1) \tau} \int_{\Omega} \bar{u}(x, t) \Psi_{1}(x, t) \mathrm{d} x \mathrm{~d} t<0 .
\end{aligned}
$$

It implies that $\left(\int_{\Omega} \bar{u}\left(x, n \tau^{+}\right) \Psi_{1}\left(x, 0^{+}\right) \mathrm{d} x\right)_{n}$ is a decreasing and non-negative sequence. Consequently, it is convergent. Moreover, we may deduce that

$$
\int_{n \tau}^{(n+1) \tau} \int_{\Omega} \bar{u}(x, t) \Psi_{1}(x, t) \mathrm{d} x \mathrm{~d} t \rightarrow 0 .
$$

Thus,

$$
\bar{u}(t) \rightarrow 0 \text { in } L^{1}(\Omega),
$$

as $t \rightarrow+\infty$. The rate of convergence is that of $e^{-\lambda_{1} t}$.

Since $u$ is the solution to (3.5), we conclude that $\bar{u}(t) \rightarrow 0$ in $L^{\infty}(\Omega)$, as $t \rightarrow+\infty$, and so, the same conclusion follows for $u$.

Remark 3.1. If we consider the space independent case, by direct calculation it follows that condition $\lambda_{1}>0$ is equivalent to $\rho e^{r \tau}<1$ and condition $\lambda_{1} \geq 0$ is equivalent to $\rho e^{r \tau} \leq 1$, which is same as the Corollary 3.1 of [24].

Theorem 3.2. Suppose $u_{0}$ satisfies (H2), if $\lambda_{1}<0$, the solution $\hat{u}$ to (3.1) satisfies

$$
\hat{u}(t) \rightarrow u^{*}(t), \quad \text { in } L^{\infty}(\Omega) \text { as } t \rightarrow+\infty,
$$

where $u^{*}(x, t)$ is the extension by $\tau$-periodicity of the unique non-negative solution to

$$
\begin{cases}\frac{\partial u}{\partial t}-d_{1} \frac{\partial^{2} u}{\partial x^{2}}=r u\left(1-\frac{u}{K}\right), & x \in \Omega, t \in(0, \tau), \\ u\left(x, 0^{+}\right)=\rho(x) u(x, \tau), & x \in \Omega, \\ u_{x}=0, & x \in \partial \Omega, t \in(0, \tau) .\end{cases}
$$


Proof. If $\lambda_{1}<0$, we can deduce that $\bar{\varphi}:=\epsilon \varphi_{1}$ is subsolution of (3.6) for all $0<\epsilon \leq-\frac{\lambda_{1} K}{r c_{1}}$, where $\varphi_{1}(x, t)$ is the principal eigenvector of (3.2), and satisfies

$$
\varphi_{1}(x, t) \geq c_{1}>0,(x, t) \in \Omega \times(0, \tau) .
$$

On the other hand, since $\rho(x) \leq 1$, we can see that $\varrho K$ is the supersolution of (3.6) for all $\varrho>1$. Thanks to comparison principle, we can get a unique non-negative solution $u^{*}(x, t)$. Moreover we can choose $\epsilon \varphi_{1}(x, 0)$ as small and $\varrho K$ as large as we please, Applying the iteration scheme argument, we can get the global attractiveness of $u^{*}$ (with respect to positive initial conditions $u_{0}(x)$ ).

\section{Single species natural enemies model}

Firstly, let us consider the following auxiliary problem:

$$
\begin{cases}\frac{\partial v}{\partial t}=d_{2} \frac{\partial^{2} v}{\partial x^{2}}-\alpha v, & x \in \Omega, t \in R^{+} \backslash n \tau, \\ v\left(x, n \tau^{+}\right)=\rho_{0}(x) v(x, n \tau)+I(x), & x \in \Omega, n \in N, \\ v_{x}=0, & x \in \partial \Omega, t \in R^{+} \backslash n \tau, \\ v(x, 0)=v_{0}(x), & x \in \partial \Omega,\end{cases}
$$

where $v_{n}(x, t)$ represents the density of the natural enemies population during period $n$, $v_{n, \tau}(x)$ denotes the density of the natural enemies population at time $n \tau$, and $v_{0}=v_{0, \tau}(x)$. By calculation, the solution of the auxiliary problem (4.1) is expressed as

$$
\hat{v}_{n}(x, t)=\int_{\Omega} G(\xi, x, t-(n-1) \tau)\left(\rho_{0} v_{n-1, \tau}+I\right)(\xi) \mathrm{d} \xi,
$$

for all $t \in((n-1) \tau, n \tau]$, where

$$
G(\xi, x, t)=\frac{2}{l} \sum_{k=1}^{\infty} e^{-\left[d_{2}\left(\frac{k \pi}{l}\right)^{2}+\alpha\right] t} \cos \frac{k \pi}{l} x \cos \frac{k \pi}{l} \xi .
$$

We assume $v_{0}(x), I(x) \in L^{\infty}(\Omega)$, then

$$
\left\|v_{1, \tau}(x)\right\|_{\infty} \leq \frac{1}{e^{\alpha \tau} \sqrt{d_{2} \pi \tau}}\left\|\int_{\Omega}\left(\rho_{0} v_{0}+I\right)(\xi) \mathrm{d} \xi\right\|_{\infty} \leq \frac{l}{e^{\alpha \tau} \sqrt{d_{2} \pi \tau}}\left(\left\|\rho_{0} v_{0}\right\|_{\infty}+\|I\|_{\infty}\right),
$$

so $v_{1, \tau}(x) \in L^{\infty}(\Omega)$, and so on $v_{n, \tau}(x) \in L^{\infty}(\Omega), n \in N$.

For any $m>n \in N$,

$$
\left\|v_{m, \tau}-v_{n, \tau}\right\|_{\infty} \leq \frac{l}{e^{\alpha \tau} \sqrt{d_{2} \pi \tau}}\left\|\rho_{0}\left(v_{m-1, \tau}-v_{n-1, \tau}\right)\right\|_{\infty}
$$




$$
\begin{aligned}
& \leq \frac{l \rho_{0}^{*}}{e^{\alpha \tau} \sqrt{d_{2} \pi \tau}}\left\|v_{m-1, \tau}-v_{n-1, \tau}\right\|_{\infty} \\
& \leq \cdots \\
& \leq\left(\frac{l \rho_{0}^{*}}{e^{\alpha \tau} \sqrt{d_{2} \pi \tau}}\right)^{n}\left\|v_{m-n, \tau}-v_{0}\right\|_{\infty} .
\end{aligned}
$$

Consequently, if $\frac{l \rho_{0}^{*}}{e^{\alpha \tau} \sqrt{d_{2} \pi \tau}}<1$, we can get $\left\{v_{n, \tau}\right\}$ is Cauchy sequence in $L^{\infty}(\Omega)$. Using the complete of $L^{\infty}(\Omega)$, there is a unique $v^{*}(x) \in L^{\infty}(\Omega)$ satisfying

$$
v_{n, \tau} \rightarrow v^{*}(x) \text {, in } L^{\infty}(\Omega)
$$

as $n \rightarrow \infty$, and

$$
v^{*}(x)=\int_{\Omega} G(\xi, x, \tau)\left(\rho_{0} v^{*}+I\right)(\xi) \mathrm{d} \xi
$$

Now, we construct equation

$$
\begin{cases}\frac{\partial v}{\partial t}=d_{2} \frac{\partial^{2} v}{\partial x^{2}}-\alpha v, & x \in \Omega, t \in R^{+} \backslash n \tau, \\ v\left(x, n \tau^{+}\right)=\rho_{0}(x) v(x, n \tau)+I(x), & x \in \Omega, n \in N, \\ v_{x}=0, & x \in \partial \Omega, t \in R^{+} \backslash n \tau, \\ v(x, 0)=v^{*}(x), & x \in \partial \Omega .\end{cases}
$$

Thus, there is a unique non-negative $\tau$-periodicity solution $\bar{v}(x, t)$, and

$$
\bar{v}(x, t)=\int_{\Omega} G\left(\xi, x, t-\left[\frac{t}{\tau}\right] \tau\right)\left(\rho_{0} v^{*}+I\right)(\xi) \mathrm{d} \xi, \quad t \in([t / \tau] \tau,([t / \tau]+1) \tau] .
$$

Then we get below Lemma.

Lemma 4.1. If $\frac{l \rho_{0}^{*}}{e^{\alpha \tau} \sqrt{d_{2} \pi \tau}}<1$, then the solution $\hat{v}_{n}(x, t)$ to (4.1) satisfies

$$
\hat{v}_{n}(t)-\bar{v}(t) \rightarrow 0 \text { in } L^{\infty}(\Omega)
$$

as $t \rightarrow+\infty$.

Remark 4.1. If we consider the space independent case, then problem (4.1) becomes

$$
\begin{cases}\frac{\mathrm{d} v}{\mathrm{~d} t}=-\alpha v, & t \in R^{+} \backslash n \tau, \\ v\left(n \tau^{+}\right)=\rho_{0} v(n \tau)+I, & n \in N, \\ v(0)=v_{0} . & \end{cases}
$$

We can get the following result by direct calculation,

$$
\hat{v}(t)=e^{-\alpha t}\left\{v_{0} \rho_{0}^{n} e^{-\alpha \tau(n-1)}+I \frac{1-\left(\rho_{0} e^{-\alpha \tau}\right)^{n}}{1-\rho_{0} e^{-\alpha \tau}}\right\}, \quad t \in((n-1) \tau, n \tau], n \in N,
$$

where $\hat{v}(t)$ is the solution to problem (4.3). 
Corollary 4.1. If $\rho_{0} e^{-\alpha \tau}<1$, the solution $\hat{v}(t)$ to (4.3) satisfies

$$
\hat{v}(t)-\bar{v}(t) \rightarrow 0
$$

as $t \rightarrow+\infty$, where $\bar{v}(t)=\frac{I}{1-\rho_{0} e^{-\alpha \tau}} e^{-\alpha t}$.

On the other hand, If $\rho_{0} e^{-\alpha \tau} \geq 1$, the solution $\hat{v}(t)$ to (4.3) satisfies

$$
\hat{v}(t) \rightarrow+\infty, \quad \text { as } t \rightarrow+\infty \text {. }
$$

\section{The boundary periodicity solution of the two species model}

Firstly, the two eigenvalue problems are considered as follow

$$
\begin{aligned}
& \begin{cases}\frac{\partial \varphi}{\partial t}-d_{1} \frac{\partial^{2} \varphi}{\partial x^{2}}-r \varphi+\beta \bar{v}(x, t) \varphi=\lambda \varphi, & x \in \Omega, t \in(0, \tau), \\
\varphi(x, 0)=\rho(x) \varphi(x, \tau), & x \in \Omega, \\
\varphi_{x}=0, & x \in \partial \Omega, t \in(0, \tau) .\end{cases} \\
& \begin{cases}\frac{\partial \varphi}{\partial t}-d_{1} \frac{\partial^{2} \varphi}{\partial x^{2}}-r \varphi+\frac{\beta \bar{v}(x, t)}{1+\beta h u^{*}(x, t)} \varphi=\lambda \varphi, & x \in \Omega, t \in(0, \tau), \\
\varphi(x, 0)=\rho(x) \varphi(x, \tau), & x \in \Omega, \\
\varphi_{x}=0, & x \in \partial \Omega, t \in(0, \tau),\end{cases}
\end{aligned}
$$

where $u^{*}(x, t)$ is the extension by $\tau$-periodicity of the unique non-negative solution to

$$
\begin{cases}\frac{\partial u}{\partial t}-d_{1} \frac{\partial^{2} u}{\partial x^{2}}=r u\left(1-\frac{u}{K}\right), & x \in \Omega, t \in(0, \tau), \\ u\left(x, 0^{+}\right)=\rho(x) u(x, \tau), & x \in \Omega, \\ u_{x}=0, & x \in \partial \Omega, t \in(0, \tau) .\end{cases}
$$

Suppose $\mu_{1}, \kappa_{1}$ be the principal eigenvalue to (5.1) and (5.2),respectively. It can be concluded that $\kappa_{1}<\mu_{1}$. we can deduce the following result.

Theorem 5.1. Suppose $u_{0}$ and $v_{0}$ satisfying $(\mathrm{H} 2)$ and $\frac{l \rho_{0}^{*}}{e^{\alpha \tau} \sqrt{d_{2} \pi \tau}}<1, \lambda_{1}<0$, (i) the solution $(u, v)$ to $(2.1)$ satisfies:

$$
\lim _{t \rightarrow+\infty} u(t)=0, \text { in } L^{\infty}(\Omega)
$$

then $\mu_{1} \geq 0$.

(ii) if $\kappa_{1}>0$, then $(u(t), v(t)) \rightarrow(0, \bar{v}(t))$ in $L^{\infty}(\Omega)$, as $t \rightarrow+\infty$. 
Proof. (i) Suppose $u(t) \rightarrow 0$ in $L^{\infty}(\Omega)$, as $t \rightarrow+\infty$. Then, for any small $\varepsilon>0$, we can get

$$
\frac{l \rho_{0}^{*}}{e^{(\alpha-\varepsilon) \tau} \sqrt{d_{2} \pi \tau}}<1
$$

and also there exists $N(\varepsilon) \in N$, such that

$$
\frac{r}{K} u(x, t)<\varepsilon, \quad \text { and } \eta \frac{\beta u(x, t)}{1+\beta h u(x, t)}<\varepsilon, \quad \text { a.e. } x \in \Omega, \forall t \geq N(\varepsilon) \tau .
$$

If $\frac{l \rho_{0}^{*}}{e^{(\alpha-\varepsilon) \tau} \sqrt{d_{2} \pi \tau}}<1$, we can get $\bar{v}_{\mathcal{\varepsilon}}(x, t)+\varepsilon \geq \tilde{v}_{\mathcal{\varepsilon}}(x, t) \geq v(x, t) \geq 0$, in $\Omega \times[N(\varepsilon) \tau,+\infty)$, where $\bar{v}_{\mathcal{\varepsilon}}$ is the solution to

$$
\begin{cases}\frac{\partial \bar{v}(x, t)}{\partial t}=d_{2} \frac{\partial^{2} \bar{v}(x, t)}{\partial x^{2}}-\alpha \bar{v}(x, t)+\varepsilon \bar{v}(x, t), & x \in \Omega, t \geq N(\varepsilon) \tau, \\ \bar{v}\left(x, n \tau^{+}\right)=\bar{v}(x, n \tau)+I(x), & x \in \Omega, n \geq N(\varepsilon), \\ \bar{v}_{x}=0, & x \in \partial \Omega, t \geq N(\varepsilon) \tau, \\ \bar{v}(x, N(\varepsilon))=v^{*}(x), & \end{cases}
$$

and $\tilde{v}_{\varepsilon}$ is the solution to

$$
\begin{cases}\frac{\partial \tilde{v}(x, t)}{\partial t}=d_{2} \frac{\partial^{2} \tilde{v}(x, t)}{\partial x^{2}}-\alpha \tilde{v}(x, t)+\varepsilon \tilde{v}(x, t), & x \in \Omega, t \geq N(\varepsilon) \tau \\ \tilde{v}\left(x, n \tau^{+}\right)=\tilde{v}(x, n \tau)+I(x), & x \in \Omega, n \geq N(\varepsilon), \\ \tilde{v}_{x}=0, & x \in \partial \Omega, t \geq N(\varepsilon) \tau \\ \tilde{v}(x, N(\varepsilon))=v(x, N(\varepsilon)) . & \end{cases}
$$

We get that

$$
\bar{v}_{\varepsilon}(x, t)-\bar{v}(x, t) \rightarrow 0 \quad \text { as } \varepsilon \rightarrow 0 .
$$

We may infer that for $t \geq N(\varepsilon) \tau$ :

$$
-\frac{\beta v(x, t)}{1+\beta h u(x, t)}>-\beta v(x, t)>-\beta\left(\bar{v}_{\mathcal{\varepsilon}}(x, t)+\varepsilon\right), \quad \text { a.e. } x \in \Omega .
$$

In conclusion,

$$
0 \leq \vec{u}(x, t) \leq u(x, t), \quad \text { a.e. } x \in \Omega, \forall t \geq N(\varepsilon),
$$

where $\vec{u}(x, t)$ is the solution to

$$
\begin{cases}\frac{\partial \vec{u}(x, t)}{\partial t}=d_{1} \frac{\partial^{2} \vec{u}(x, t)}{\partial x^{2}}+(r-\varepsilon) \vec{u}(x, t)-r \frac{\vec{u}^{2}(x, t)}{K} & \\ \quad-\beta\left(\bar{v}_{\varepsilon}(x, t)+\varepsilon\right) \vec{u}(x, t), & x \in \Omega, t \geq N(\varepsilon) \tau, \\ \vec{u}\left(x, n \tau^{+}\right)=\rho(x) \vec{u}(x, n \tau), & x \in \Omega, n \geq N(\varepsilon), \\ \vec{u}_{x}=0, & x \in \partial \Omega, t \geq N(\varepsilon) \tau, \\ \vec{u}\left(x, N(\varepsilon) \tau^{+}\right)=u\left(x, N(\varepsilon) \tau^{+}\right), & x \in \partial \Omega .\end{cases}
$$


Since $u(t) \rightarrow 0$ in $L^{\infty}(\Omega)$, as $t \rightarrow+\infty$, we conclude that $\vec{u}(t) \rightarrow 0$ in $L^{\infty}(\Omega)$, as $t \rightarrow+\infty$ and in the same manner as in Theorem 3.1 we get that

$$
\mu_{1 \varepsilon}>0,
$$

where $\mu_{1 \varepsilon}>0$ is the principal eigenvalue to (4.3), corresponding to $r:=r-\varepsilon, \bar{v}(x, t):=$ $\bar{v}_{\varepsilon}(x, t)+\varepsilon, \mu_{1}:=\mu_{1 \varepsilon}$. Since $\lim _{\varepsilon \rightarrow 0} \mu_{1 \varepsilon}=\mu_{1}$, then $\mu_{1} \geq 0$.

(ii) Suppose that $\kappa_{1}>0$. If $\lambda_{1}<0$, Using a comparison result (see [27]), we get that there exists $N_{1}(\varepsilon) \in N$, such that

$$
u^{*}(x, t) \geq u(x, t), \quad \bar{v}(x, t)-\varepsilon \leq \hat{v}(x, t) \leq v(x, t), \quad(x, t) \in \Omega \times\left[N_{1}(\varepsilon) \tau,+\infty\right)
$$

for arbitrary $\varepsilon>0$, where $u^{*}(x, t)$ is the extension by $\tau$-periodicity of the unique nonnegative solution to (5.3), $\hat{v}(x, t)$ is the solution of $(3.6), \bar{v}(x, t)$ is the solution of (4.1). then

$$
-\frac{\beta(\bar{v}(x, t)-\varepsilon)}{1+\beta h u^{*}(x, t)} \geq-\frac{\beta v(x, t)}{1+\beta h u(x, t)}
$$

and so

$$
U(x, t) \geq u(x, t), \quad(x, t) \in \Omega \times[0,+\infty),
$$

where $U(x, t)$ is the solution of

$$
\begin{cases}\frac{\partial u}{\partial t}-d_{1} \frac{\partial^{2} u}{\partial x^{2}}=r u\left(1-\frac{u}{K}\right)-\frac{\beta(\bar{v}(x, t)-\varepsilon)}{1+\beta h u^{*}(x, t)} u, & x \in \Omega, t \in R^{+} \backslash n \tau, \\ u\left(x, n \tau^{+}\right)=\rho(x) u(x, n \tau), & x \in \Omega, \\ u_{x}=0, & x \in \partial \Omega, t \in R^{+} \backslash n \tau, \\ u(x, 0)=u_{0}, & x \in \partial \Omega .\end{cases}
$$

We can get

$$
\lim _{\varepsilon \rightarrow 0} \kappa_{1 \varepsilon}=\kappa_{1},
$$

where $\kappa_{1 \varepsilon}$ is the the principal eigenvalue to

$$
\begin{cases}\frac{\partial \varphi}{\partial t}-d_{1} \frac{\partial^{2} \varphi}{\partial x^{2}}-r \varphi+\frac{\beta(\bar{v}(x, t)-\varepsilon)}{1+\beta h u^{*}(x, t)} \varphi=\lambda \varphi, & x \in \Omega, t \in(0, \tau), \\ \varphi(x, 0)=\rho(x) \varphi(x, \tau), & x \in \Omega, \\ \varphi_{x}=0, & x \in \partial \Omega, t \in(0, \tau) .\end{cases}
$$

Since $\kappa_{1}>0$, there exists very small $\varepsilon$ such that

$$
\kappa_{1 \varepsilon}>0 .
$$

In the same manner as in Theorem 3.1, we get that

$$
U(t) \rightarrow 0 \quad \text { in } L^{\infty}(\Omega) \text { as } t \rightarrow+\infty,
$$


and consequently

$$
u(t) \rightarrow 0 \text { in } L^{\infty}(\Omega) \text { as } t \rightarrow+\infty .
$$

Then,

$$
v(t) \rightarrow \bar{v}(t) \text { in } L^{\infty}(\Omega) \text { as } t \rightarrow+\infty .
$$

Remark 5.1. Let $M$ is the maximum of $\bar{v}(x, t),(x, t) \in \Omega \times(0,+\infty)$, then $M=\left\|\rho_{0} v^{*}+I\right\|_{\infty}$. If we assumed $\rho(x)=\rho$ is a constant, we consider the following two eigenvalue problems:

$$
\begin{aligned}
& \begin{cases}\frac{\partial \varphi}{\partial t}-d_{1} \frac{\partial^{2} \varphi}{\partial x^{2}}-r \varphi+\beta M \varphi=\lambda \varphi, & x \in \Omega, t \in(0, \tau), \\
\varphi(x, 0)=\rho \varphi(x, \tau), & x \in \Omega, \\
\varphi_{x}=0, & x \in \partial \Omega, t \in(0, \tau),\end{cases} \\
& \begin{cases}\frac{\partial \varphi}{\partial t}-d_{1} \frac{\partial^{2} \varphi}{\partial x^{2}}-r \varphi=\lambda \varphi, & x \in \Omega, t \in(0, \tau), \\
\varphi(x, 0)=\rho \varphi(x, \tau), & x \in \Omega, \\
\varphi_{x}=0, & x \in \partial \Omega, t \in(0, \tau) .\end{cases}
\end{aligned}
$$

We can get $\zeta_{1}>\kappa_{1}>\mu_{1}>v_{1}$. Let $\zeta_{1}$ be the principal eigenvalue to (5.9) and $\nu_{1}$ be the principal eigenvalue to (5.10).

We can get the following corollary.

Corollary 5.1. The eigenvalue to (5.9) and (5.10) satisfies

$$
\rho e^{\tau\left(\zeta_{k}+r-\beta M-\left(\frac{k \pi}{l}\right)^{2} d_{1}\right)}=1, \quad \rho e^{\tau\left(v_{k}+r-\left(\frac{k \pi}{l}\right)^{2} d_{1}\right)}=1 .
$$

Proof. Let $\varphi=e^{(\lambda+r-\beta M) t} w$, then problem (5.9) becomes

$$
\begin{cases}\frac{\partial w}{\partial t}-d_{1} \frac{\partial^{2} w}{\partial x^{2}}=0, & x \in \Omega, t \in(0, \tau), \\ w(x, 0)=\rho e^{(\lambda+r-\beta M) \tau} w(x, \tau), & x \in \Omega, \\ w_{x}=0, & x \in \partial \Omega, t \in(0, \tau) .\end{cases}
$$

Using the method of separation of variables, we can get

$$
w(x, t)=\sum_{k=1}^{+\infty} A_{k} e^{-\left(\frac{k \pi}{T}\right)^{2} d_{1} t} \sin \frac{k \pi}{l} x, \quad x \in \Omega, t \in(0, \tau) .
$$

Use the condition $w(x, 0)=\rho e^{(\lambda+r-\beta M) \tau} w(x, \tau)$, we can get

$$
\sum_{k=1}^{+\infty} A_{k}\left(1-\rho e^{\tau\left(\lambda+r-\beta M-\left(\frac{k \pi}{T}\right)^{2} d_{1}\right)}\right) \sin \frac{k \pi}{l} x=0 .
$$


Multiply both sides with $\sin \frac{k \pi}{l} x$, and integral on $(0, l)$, we can get

$$
1-\rho e^{\tau\left(\lambda+r-\beta M-\left(\frac{k \pi}{l}\right)^{2} d_{1}\right)}=0
$$

for all $k \in N$. So, $\zeta_{k}$ satisfies $1-\rho e^{\tau\left(\zeta_{k}+r-\beta M-\left(\frac{k \pi}{l}\right)^{2} d_{1}\right)}=0$. Also, we can get $1-\rho e^{\tau\left(v_{k}+r-\left(\frac{k \pi}{l}\right)^{2} d_{1}\right)}=$ 0 for the same methods.

According to Theorem 4.1, we can get the following corollary.

Corollary 5.2. Assume that $\frac{l \rho_{0}^{*}}{e^{\alpha \tau} \sqrt{d_{2} \pi \tau}}<1$. If $\rho(x)=\rho$ is a constant and for any $u_{0}$ and $v_{0}$ satisfying $(\mathrm{H} 2)$, the solution $(u, v)$ to $(2.1)$ satisfies:

$$
\lim _{t \rightarrow+\infty} u(t)=0
$$

in $L^{\infty}(\Omega)$, then

$$
\rho e^{\tau\left(r-\beta M-\frac{\pi^{2}}{l^{2}} d_{1}\right)}<1
$$

On the other hand, if

$$
\rho e^{\tau\left(r-\frac{\pi^{2}}{l^{2}} d_{1}\right)}<1,
$$

then for any $u_{0}$ and $v_{0}$ satisfying $(\mathrm{H} 2), u(t) \rightarrow 0$ in $L^{\infty}(\Omega)$, as $t \rightarrow+\infty$.

Remark 5.2. If we consider the space independent case, then system (2.1) becomes

$$
\begin{cases}\frac{\mathrm{d} u}{\mathrm{~d} t}=r u\left(1-\frac{u}{K}\right)-\frac{\beta u v}{1+\beta h u^{\prime}} & t \in R^{+} \backslash n \tau, \\ \frac{\mathrm{d} v}{\mathrm{~d} t}=-\alpha v+\frac{\eta \beta u v}{1+\beta h u^{\prime}}, & t \in R^{+} \backslash n \tau, \\ u\left(n \tau^{+}\right)=\rho u(n \tau), & n \in N, \\ v\left(n \tau^{+}\right)=\rho_{0} v(n \tau)+I, & n \in N, \\ u(0)=u_{0}, \quad v(0)=v_{0} . & \end{cases}
$$

Finally, we obtain the following corollary.

Corollary 5.3. If $\rho_{0} e^{-\alpha \tau}<1$, for any $u_{0}$ and $v_{0}$ satisfying $(\mathrm{H} 2)$, the solution $(u, v)$ to (5.12) satisfies:

$$
\lim _{t \rightarrow+\infty} u(t)=0
$$

then

$$
\rho e^{r \tau-\beta \int_{0}^{\tau} \bar{v}(t) \mathrm{d} t} \leq 1
$$

Since $\bar{v}(t)=\frac{I}{1-\rho_{0} e^{-\alpha \tau}} e^{-\alpha t}$, this condition is equivalent to $\rho e^{r \tau-\beta \frac{1-e^{-\alpha \tau}}{\alpha\left(1-\rho_{0} e^{e \tau}\right)}} I \leq 1$. 
On the other hand, if

$$
\rho e^{r \tau-\frac{\beta}{1+\beta h K} \int_{0}^{\tau} \bar{v}(t) \mathrm{d} t}<1
$$

or

$$
\rho e^{r \tau-\frac{\beta}{1+\beta h K} \frac{1-e^{-\alpha \tau}}{\alpha\left(1-\rho_{0} e^{\alpha \tau}\right)} I}<1,
$$

then for any $u_{0}$ and $v_{0}$ satisfying $(\mathrm{H} 2)$, we have that $u(t) \rightarrow 0$, as $t \rightarrow+\infty$, where $(u, v)$ is the solution to (5.12).

\section{Simulations for the models}

In order to verify the validity of the results, we consider system (2.1) with $d_{1}=d_{2}=r=K=$ $1, \tau=2, \beta=h=\alpha=\eta=1$. Choose $\rho=0.12, \rho_{0}=0.8$, obviously, we have that the parameters satisfy the condition of Corollary 5.2,

$$
\frac{l \rho_{0}}{e^{\alpha \tau} \sqrt{d_{2} \pi \tau}}=0.8889<1,
$$

and

$$
\rho e^{\tau\left(r-\frac{\pi^{2}}{l^{2}} d_{1}\right)}=0.844<1 .
$$

See Fig. 1. Here we choose $x \in \Omega=(0,20)$ and the initial condition $u_{0}(x)=v_{0}(x)=e^{-(x-10)^{2}}$, the natural enemy release function $I(x)=0.1$ for all $x \in \Omega$. In this case, pests are extinct and natural enemies have a positive periodic solution.

If we choose $\rho=0.12, \rho_{0}=0.95$, we have that the parameters satisfy the condition:

$$
\frac{l \rho_{0}}{e^{\alpha \tau} \sqrt{d_{2} \pi \tau}}=1.0258>1,
$$

and

$$
\rho e^{\tau\left(r-\frac{\pi^{2}}{l^{2}} d_{1}\right)}=0.844<1 .
$$

By numerical simulation, we can get the similar figure with Fig. 1. Here we give Fig. 2. From Fig. 2, pests also are extinct and natural enemies also have a positive periodic solution. This means that the conditions in 5.2 are sufficient conditions, not necessary.

If we choose $\rho=0.5, \rho_{0}=0.8$, we have that the parameters satisfy the condition:

$$
\frac{l \rho_{0}}{e^{\alpha \tau} \sqrt{d_{2} \pi \tau}}=0.8889<1,
$$

and

$$
\rho e^{\tau\left(r-\frac{\pi^{2}}{l^{2}} d_{1}\right)}=3.5166>1 .
$$

Form Fig. 3, pests and predators can coexist, all have positive periodic solutions. 

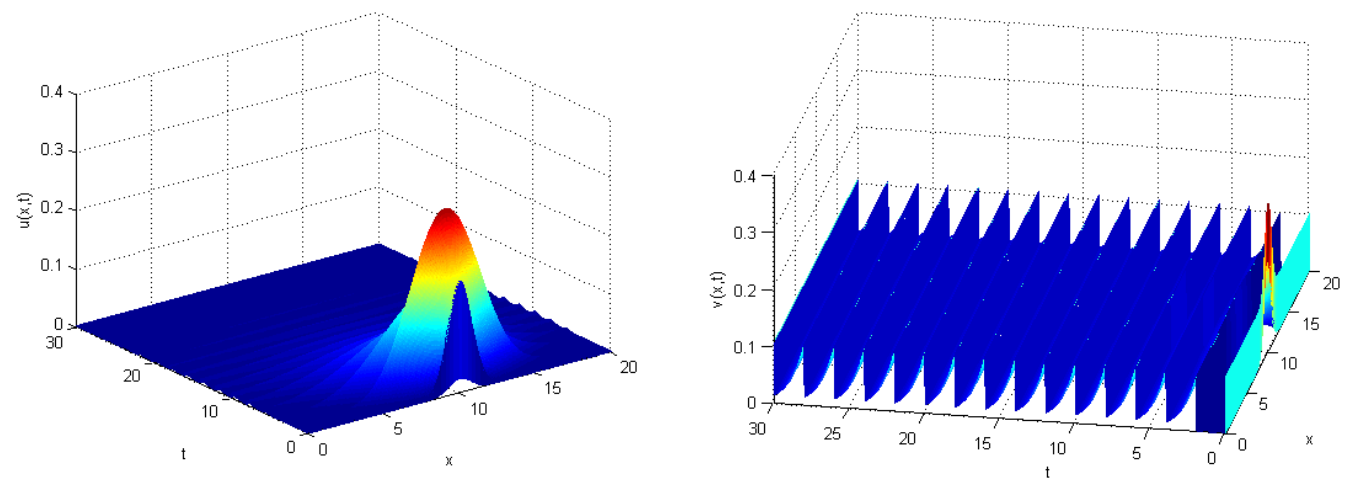

Figure 1: A numerical approximation to $u$ and $v$ with $\rho=0.12, \rho_{0}=0.8$.
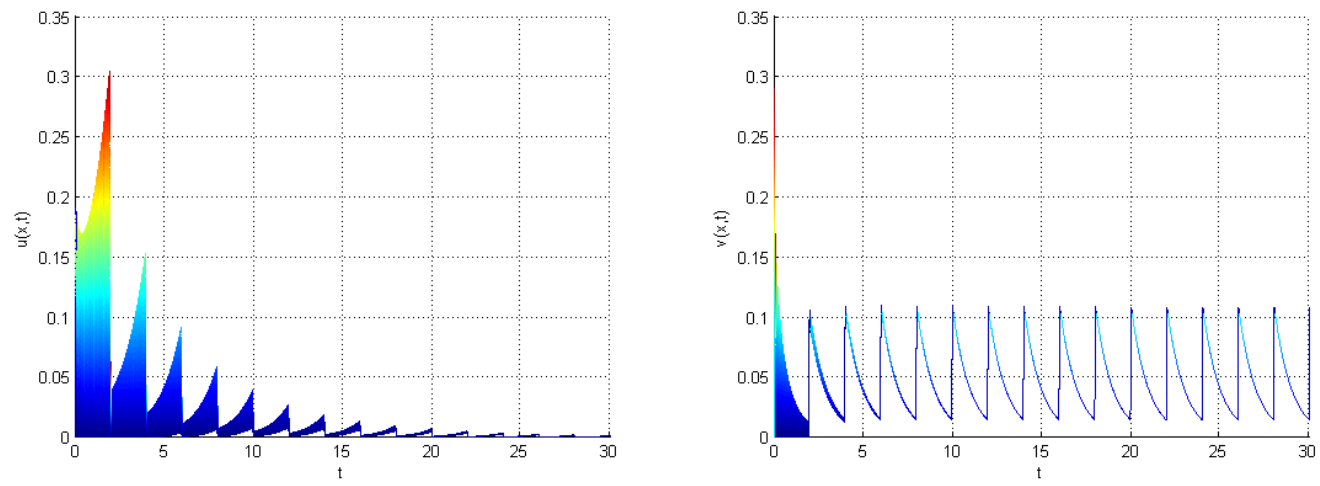

Figure 2: A numerical approximation to $u$ and $v$ at $x=0$ with $\rho=0.12, \rho_{0}=0.95$.

\section{Conclusion and discussion}

In this article, we assume that the released natural enemies have less ability to adapt to new environment (intrinsic growth rate is less than zero) and uneven distribution of space (for convenience, assuming one dimensional space). Moreover, based on the control strategy of pest removal, the control strategy of natural enemies is added. At this point, the model is more reasonable and more applicable. It is found that the existence of the positive periodic solution of the single population pulse of natural enemy system is dependent of the release function $I(x)$, it is found that the increase of the release function $I(x)$ will cause the increase of the positive periodic solution. Meanwhile, the increase of $I(x)$ will indirectly make the condition of pest extinction easier to reach, which is straightforward to observe when the spatial distribution is uniform (Corollary 5.3). In addition, due to the complexity of partial differential equations, we do not find the explicit conditions for the extinction of pests, but when the survival rate of pests is constant, we get the 

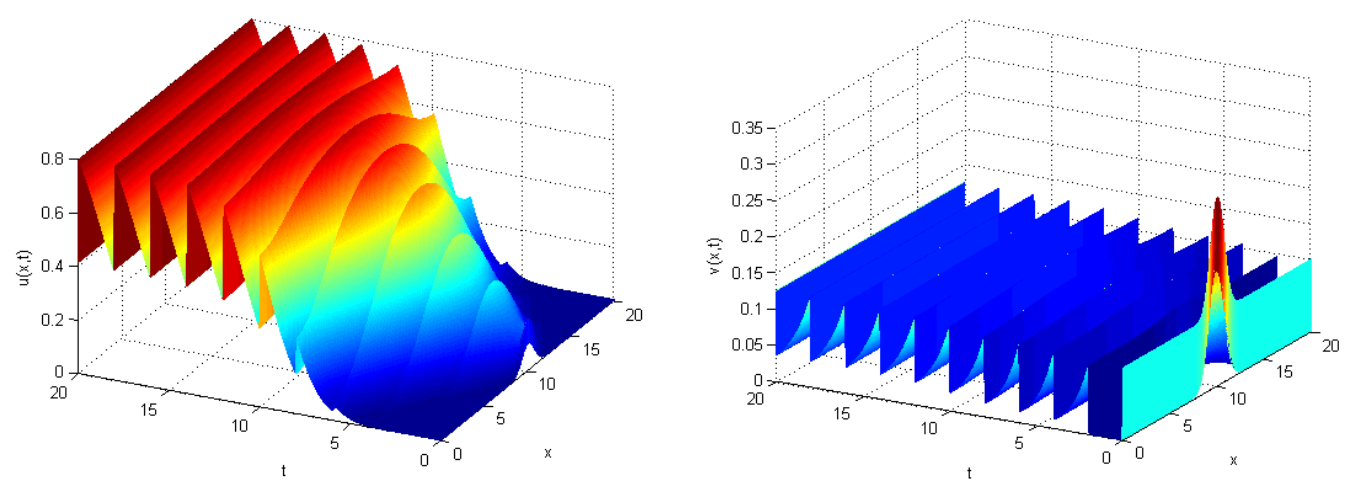

Figure 3: A numerical approximation to $u$ and $v$ with $\rho=0.5, \rho_{0}=0.8$.

explicit sufficient condition and necessary conditions for the extinction of pests, which makes our conclusions more practical (Corollary5.2).

Functional response of natural enemies and boundary condition

We use Holling II type functional response to describe the interaction between pest and natural enemies, when the handing time equals zero, the functional response is the simplest form. Moreover, the functional response can also be changed to Holling III and $\mathrm{IV}$, and the conclusions are similar.

We assume that the second type of boundary condition holds for the pest and natural enemy population, model with the third types of boundary can also be discussed with the same methods.

The direction of later research

In the model, pulse control occurs which involves release of natural enemies and spraying pesticides in a fixed time. In fact, it can be improved to other two forms:

(a) spraying pesticides more(less) frequently than releasing natural enemies;

(b) releasing natural enemies and spraying pesticides only when pest densities reach their economic threshold (ET) [16]. And all of these can be used as a direction for later research.

\section{Acknowledgement}

The author wishes to express their gratitude to the editors and the reviewers for the helpful comments. The work is supported by the NSFC $(11831003,11771031,11531010)$ of China, NSF of Qinghai Province (2017-ZJ-908), the young backbone teachers project of undergraduate universities of Henan Province in 2021, the science and technology key project of Henan Province of China under Grants $(212102310464,182102110292)$ and Key Scientific Research Project of Higher Education Institutions of Henan Province (21A110015). 


\section{References}

[1] Flint M. L., Integrated Pest Management for Walnuts. University of California, Oakland, CA, 1987.

[2] Lenteren J. V., Integrated pest management in protected crops. Integrated Pest Management D., 17 (3) (1995), 270-275.

[3] Lenteren J. V., Woets J., Biological and integrated pest control in greenhouses. Annual Review of Entomology, 33 (1988), 239-269.

[4] Lenteren J. V., Success in Biological Control of Arthropods by Augmentation of Natural Enemies. Springer Netherlands, 2000.

[5] Hoffmann M. P., Frodsham A., Natural enemies of vegetable insect pests. Florida Entomologist, 76 (3) (1993), 531-541.

[6] Parker F. D., Management of Pest Populations by Manipulating Densities of Both Hosts and Parasites through Periodic Releases. Springer US, 1971.

[7] Liang J., Adaptive release of natural enemies in a pest-natural enemy system with pesticide resistance. Bull. Math. Biol., 75 (11) (2013), 2167-2195.

[8] Neuenschwander P., et al., Impact assessment of the biological control of the cassava mealybug, phenacoccus manihoti matile-ferrero (hemiptera: Pseudococcidae), by the introduced parasitoid epidinocarsis lopezi (de santis) (hymenoptera: Encyrtidae). Bulletin of Entomological Research, 79 (4) (1989), 579-594.

[9] Tang S., et al., Integrated pest management models and their dynamical behaviour. Bull. Math. Biol., 67 (1) (2005), 115-135.

[10] Liang J., et al., Optimal dosage and economic threshold of multiple pesticide applications for pest control. Math. Comput. Modelling, 51 (5-6) (2010), 487-503.

[11] Tang S., et al., State-dependent impulsive models of integrated pest management (ipm) strategies and their dynamic consequences. J. Math. Biol., 50 (3) (2005), 257-292.

[12] Tang S., et al., Multiple attractors of host-parasitoid models with integrated pest management strategies: Eradication, persistence and outbreak. Theoret. Population Biol., 73 (2) (2008), 181-197.

[13] Tang S., et al., Models for integrated pest control and their biological implications. Math. Biosci., 215 (1) (2008), 115-125.

[14] Tang S., et al., Effects of predator and prey dispersal on success or failure of biological control. Bull. Math. Biol., 71 (8) (2009), 2025-2047.

[15] Tang S., et al., Optimum timing for integrated pest management: modelling rates of pesticide application and natural enemy releases. J. Theoret. Biol., 264 (2) (2010), 623-633.

[16] Tang S., et al., Threshold conditions for integrated pest management models with pesticides that have residual effects. J. Math. Biol., 66 (1-2) (2013), 1-35.

[17] Barclay H. J., Models for pest control using predator release, habitat management and pesticide release in combination. Journal of Applied Ecology, 19 (2) (1982), 337-348.

[18] Debach P., Biological Control by Natural Enemies. Cambridge University Press, Cambridge, 1991.

[19] Ruberson J., Pesticides and Conservation of Natural Enemies in Pest Management. New York: Academic Press, 1998.

[20] Bainov D. D., et al., Systems with Impulse Effect : Stability, Theory, and Applications. E. Horwood , Halsted Press, 1989.

[21] Bainov D. D., Impulsive Differential Equations: Periodic Solutions and Applications. Long- 
man Scientific and Technical, 1993.

[22] Roberts M. G., et al., The dynamics of an infectious disease in a population with birth pulses. Math. Biosci., 149 (1) (1998), 23-34.

[23] Akhmet M., et al., An impulsive ratio-dependent predator-prey system with diffusion. Nonlinear Anal. Real World Appl., 7 (5) (2006), 1255-1267.

[24] Anita S., et al., Impulsive spatial control of invading pests by generalist predators. Mathematical Medicine and Biology-a Journal of the IMA, 31 (3) (2014), 284-301.

[25] Anita S., et al., Stabilization of a reaction-diffusion system modelling a class of spatially structured epidemic systems via feedback control. Nonlinear Anal. Real World Appl., 13 (2) (2012), 725-735.

[26] Anita S., et al., A stabilization strategy for a reaction-diffusion system modelling a class of spatially structured epidemic systems ( think globally, act locally). Nonlinear Anal. Real World Appl., 10 (4) (2009), 2026-2035.

[27] Protter M. H., Maximum Principles in Differential Equations. New York: Springer, 1984. 